\title{
HIGH TEMPERATURE SUPERCONDUCTING BEARINGS FOR LUNAR TELESCOPE MOUNTS
}

\author{
Mark Lamb, Ki BuiMa, Rodger Cooley, Daniel Mackey, Ruling Meng, Ching Wu Chu and Wei Kan Chu \\ Texas Center for Superconductivity, University of Houston, Houston, TX. \\ Peter C. Chen \\ Computer Sciences Corporation, Calverton, MD \\ Thomas Wilson
Aeronautics and Space Administration, Houston, Texas \\ Thomas Wilson
Johnson Space Center, National Aeronautics and Space Administration, Houston, Texas

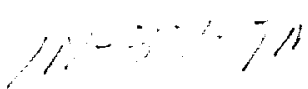

\begin{abstract}
A telescope to be installed on the lunar surface in the near future must work in a cold and dusty vacuum environment for long periods without on site human maintenance. To track stars, the drive mechanism must be capable of exceedingly fine steps and repeatability. Further, the use of lightweight telescopes for obrious economic benefits burdens the requirement for stable support and rotation. Conventional contact bearings and gear drives have numerous failure modes under such a restrictive and harsh environment. However, bybrid superconducting magnetic bearings (HSMB) fit in naturally. These bearings are stable, light, passive, and essentially frictionless, allowing high precision electronic positioning control. By passive levitation, the HSMB does not wear out and requires neither maintenance nor power. A prototype illustrating the feasability of this application is presented.
\end{abstract}

\section{INTRODUCTION}

Since the discovery of the new high temperature superconductors (HTS) in 1987, the vision of modern mechanical devices incorporating passive levitation technology has enticed scientists and engineers alike. At this time, the use of a cryogen still tends to limit many applications until more practical critical temperatures are achieved. However, if one is to operate on the moon, the current superconductors are already room temperature, and enable passive levitation technology not only to be practical but natural.

Such an opportunity to apply HTS technology presents itself in the lunar telescope as proposed by Chen [1]. A telescope on the moon is considered to be an ideal observatory with the astronomers. It must be lightweight, yet maintain high tracking resolution and repeatability, and should require no onsite maintenance for an estimated five years, a challenging if not impossible task with conventional technology. Chen proposes incorporating the following advanced technologies to overcome problems: chargeinjecting device imagers to avoid charge-coupled device's (CCD) susceptibility to radiation damage [2]; optical elements of very low areal density $\left(<2 \mathrm{~kg} / \mathrm{m}^{2}\right)$ to meet

Manuscript received October 17, 1994

This work is supported in part by the Advanced Research Project Agency and in part by the State of Texas through the Texas Center of Superconductivity. payload weight limitations [3]; and HTS bearings to meet the strict maintenance and resolution requirements which conventional bearings cannot maintain. We report on the first prototype HTS bearing module built at the University of Houston in collaboration with Chen to be assembled as an azimuth mount for a levitated lunar telescope, see Fig. 1.

\section{LUNAR BEARING REQUTREMENTS}

To track the position of a celestial object, the telescope must be able to change orientation as the moon rotates about Earth and its own axis. In general, the bearing mmule would have rotation capability in three directions.

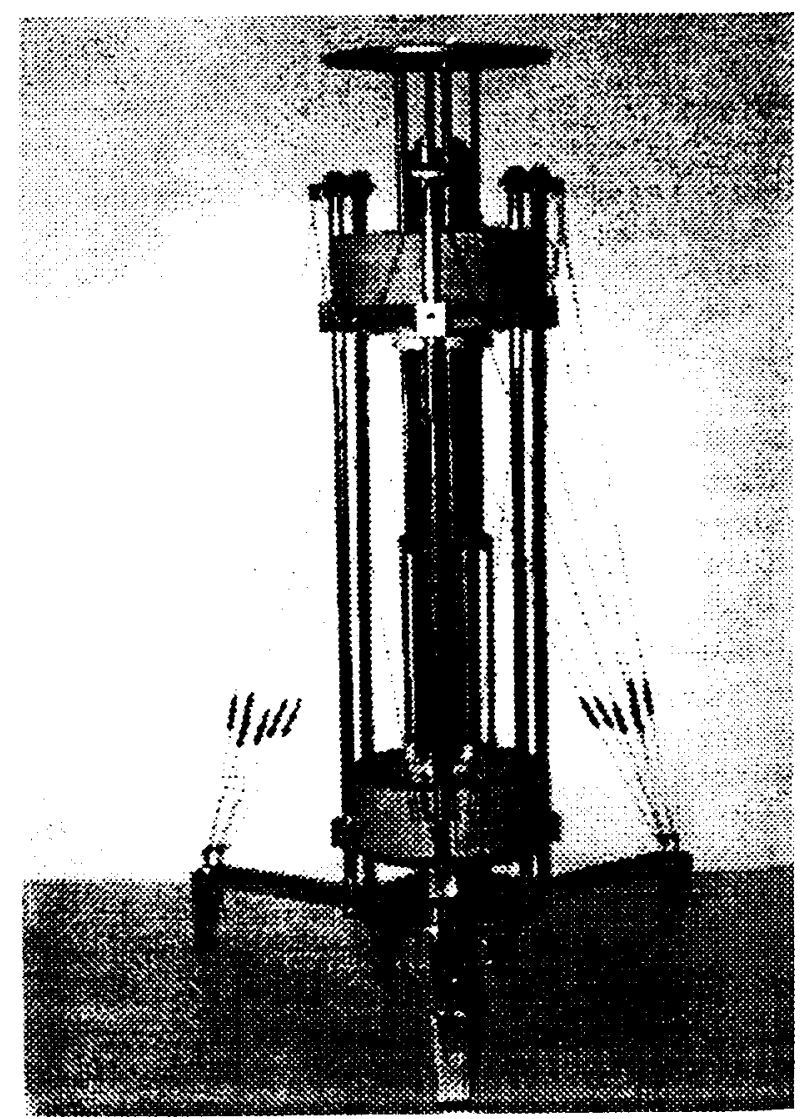

Fig. 1. The HTS bearing module. Lunar telescope to be mounted on the platform at top of levitating assembly, approximately one meter high 
The bearing module discussed here is an azimuth mount providing free rotation only about the gravitational axis. Future prototypes will allow greater degrees of freedom. The relevant performance parameter to the astronomer is the degree of steadiness the direction of the telescope maintains with the target. Steadiness is usually measured as the angle subtended by the line of deviation from the target. For observations from the moon, an angle no greater than 0.1 aresecond is desirable. Assuming the ground is not moving, the more rigid a bearing is to angular deviations from the astronomer's target, the better will be the performance. Hence, a high stiffness bearing is preferred.

The advantages over conventional bearings which levitation bearings provide for the lunar environment are the ability to operate preferably in low temperature and high vacuum and to provide extremely smooth and therefore highly controllable motion. The current conventional machinery that can achieve the kind of extremely fine motion required to track a star from the moon are massive, have small travel ranges, require carefully controlled environments, and need periodic maintenance, making them unsuitable for use on the moon. In addition, the low temperature on the lunar surface tends to exclude mechanical designs with fine and closely fitted parts due to differences in thermal contraction of the separate parts. Almost all lubricants are disqualified because of the low vacuum and low temperature.

\section{HYBRID HTS BEARINGS}

The above restrictions imposed on a lunar telescope with an HTS bearing made of the current materials, e.g. melttextured $\mathrm{YBaCuO}$, require that a hybrid levitation scheme is adapted similar to those previously reported [4]-[6]. The hybrid superconducting magnetic bearing (HSMB) technology developed for this lunar telescope utilizes an effectively zero stiffness, i.e. neutral stability, permanent magnet system to supply a thrust force to counter gravity and levitate the system. The HTS provides only positive stiffness to stabilize the levitated system.

The HSMB technology affords numerous benefits to the practical adaptation of HTS levitation applications. It is capable of overcoming the weight limitation inherent in nonhybrid designs and permits the more practical field cooled operation of the HTS. Design also is more versatile as demonstrated in Section III and is simplified because the forces between permanent magnets is easily calculated by numerical methods.

More importantly, for this application, the HSMB is necessary to reduce complications wrought by induced flux motion and the numerous phase transitions of the flux line lattice within the HTS. Under various perturbations, e.g., varying magnetic fields, displacement of the magnet from equilibrium, and changes in temperature, the HTS-magnet force can undergo a transition from an elastic to a plastic response and exhibit relaxation [7]-[9]. As a consequence, the dynamic levitation force provided by the HTS in a perturbing environment is frequently not a predictable parameter, and at the least, it is not a controllable parameter. The HSMB technology reduces the dependence of this parameter on the HTS. Zero stiffness levitation force provided by the high coercive rare earth permanent magnets, e.g. $\mathrm{NdBFe}$, is much less susceptible to various perturbations (effectively zero in the first order for the temperature and field). The dynamic mechanical stability component from the HTS system is then not burdened by the particular phase the magnetic flux lattice may be in, as long as linearization of the peculiar force produces a sufficiently high positive stiffness, even though this stiffness may vary between phases.

\section{BEARING ModUle Design}

\section{A. The Counterweight and the Lift}

The bearing module needs to levitate only a very light load, but it must maintain the telescope in an upright position above the bearing and withstand the tendency to topple. The bearing module accounts for this problem by providing an effective counterweight with magnetic forces equivalent in principle to the counterweight used by conventional telescopes on earth. A schematic of the bearing module is shown in Fig. 2. The counterweight system is a separate HSMB arranged upside down so that the force pulls down on the rotor assembly and placed low enough so that the effective center of gravity of the levitated system falls below the pivotal point of support at the lift system.

The lift system supplies only the force necessary to counter the weight of the entire rotor assembly which includes the counterweight, the lift, the platform, and the frame. By symmetrically adjusting the gap between the two $3.8 \mathrm{~cm}$ diameter supporting magnet rings, the lift force and the counterweight force each can be independently adjusted without losing the condition of neutral stability to accommodate a range of possible weights which may be attached to the platform. Because of variations in materials, the adjustment is a necessary mechanism to ease the design and fabrication constraints.

\section{B. The HTS System}

Each HSMB has a $10 \mathrm{~cm}$ diameter rotor magnet attached to the assembly which consists of two concentric ring magnets, one fitted into the other with opposite polarity. The opposite polarities were designed to maximize the stiffness from the HTS by increasing the gradient of the magnetic field that would be frozen into the superconductor. Because the rotor magnets have an opposite polarity arrangement the total thrust is the sum of two opposing thrust and consequently is not the optimal that can be obtained from the volume of magnetic material. 


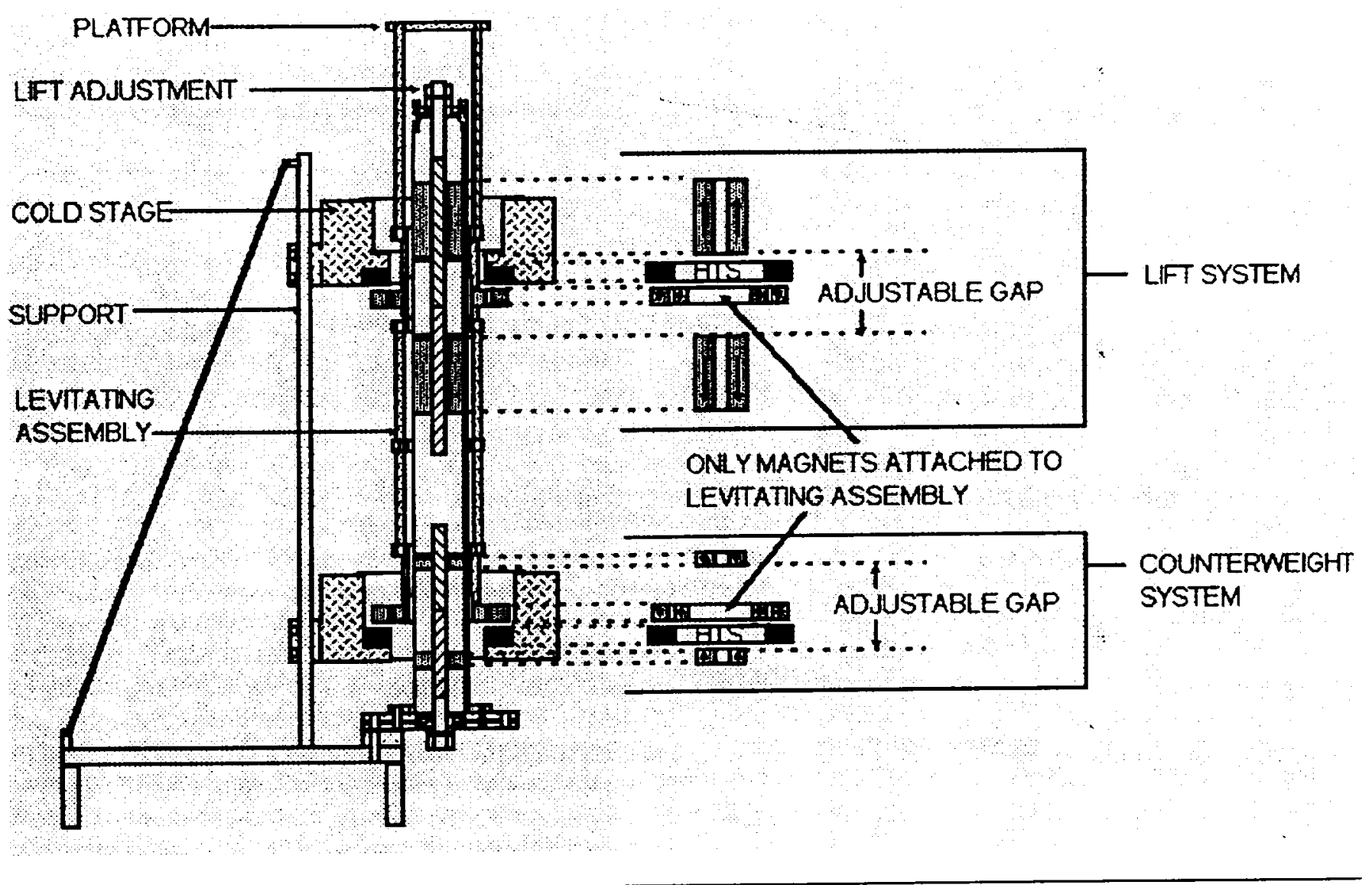

Fig. 2. Diagram of the systems and components of the bearing module.

\section{The Support Structure}

1) Inner support tube: The two stator magnets for each bearing are contained within a thin walled stainless steel tube and attached to a screw drive mechanism which symmetrically adjusts the gap between them. The screw drive for the lift system and the counterweight system is attached to an adjustment knob at the top and the bottom of the tube, respectively.

2) Outer support truss: The HTS for each bearing is fixed within a high density Styrofoam and G10 fiberglass epoxy cold stage. The cold stages are supported by a three legged tension cord truss bolted to a flange at the bottom of the inner support tube and are height adjustable. The entire bearing module has a mass just under $7 \mathrm{Kg}$.

\section{Performance of Bearing Module}

\section{A. The Zero Stiffness Magnet System}

The axial force as a function of axial displacement at various gap distances are plotted in Fig. 3 for the counterweight magnet system. The positive force on this graph represents the pull force acting down on the assembly The agreement between the experimental results after fabrication and the numerical calculation in the design phase

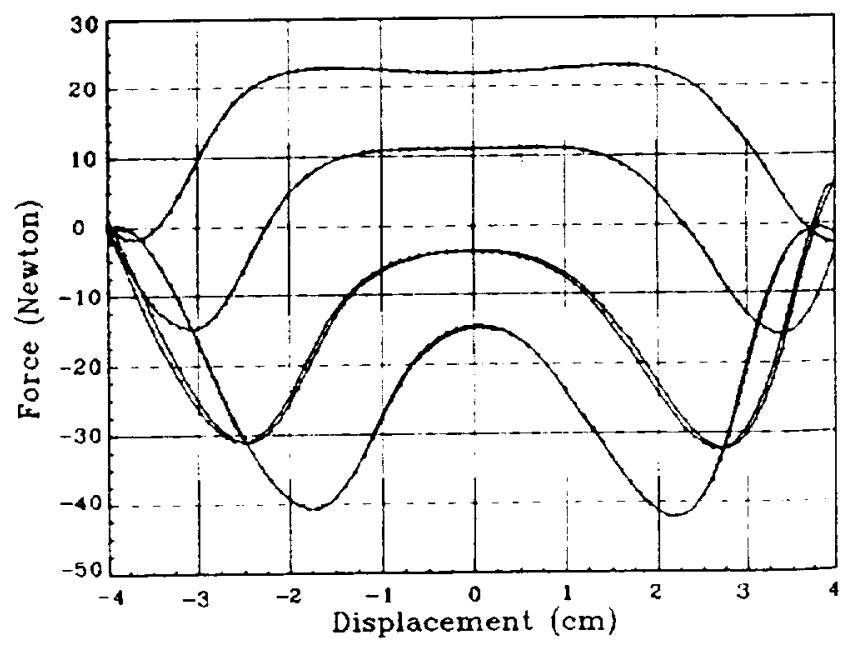

Fig. 3. Axial force vs. axial displacement for counterweight bearing magnet system at gap distances of $3.8 \mathrm{~cm}(1.5 \mathrm{in}), 5.1 \mathrm{~cm}(2.0 \mathrm{in}), 6.4 \mathrm{~cm}(2.5 \mathrm{in})$, and $7.6 \mathrm{~cm}(3.0 \mathrm{in})$ going from the bottom curve to the top curve. respectively. The zero stiffness behavior was optimized by design for the nominal force of $12.0 \mathrm{~N}$. Positive force represents the force pulling down on the assembly. 
is excellent. The counterweight magnet system is designed to provide optimum zero stiffness at a nominal force equal to the telescope's specified weight of $12.0 \mathrm{~N}$ ( $2.7 \mathrm{lbs})$. This optimization was achieved as evidenced by the curve at a gap distance of $6.4 \mathrm{~cm}$ (2.5 in), second from the top in Fig. 3, which displays a constant force over $2 \mathrm{~cm}$. The lift magnet system is similar except the range of force is greater and represents the push force acting to lift the entire assembly. The top bearing supports a nominal load of $44.6 \mathrm{~N}$ (10 lbs).

\section{B. The HTS - System Stability}

The axial force as a function of the axial separation distance between the rotor magnet and the HTS for one bearing is shown in Fig. 4. The four curves represent the force response of the bearing at four different field cooled gaps between the HTS and rotor magnet obtained by adjusting the position of the cold stage along the support truss. The HTS was field cooled from room temperature to approximately 77 degrees Kelvin by filling the cold stages with liquid nitrogen. It took about three minutes for the HTS to become superconducting.

For an initial measurement of the steadiness, the HTS gap was set arbitrarily at $8 \mathrm{~mm}$ giving a total axial stiffness of $10 \mathrm{~N} / \mathrm{mm}(60 \mathrm{lbs} / \mathrm{in})$ to the entire assembly. As can be seen by Fig. 4, a gap of $6 \mathrm{~mm}$ will double this number and, as is well known, the smaller the gap, the higher the stiffness, so that, if deemed necessary, a much higher stiffness can be obtained from this module.

\section{The Overall Steadiness}

To measure the steadiness, the module was prepared as above on a laboratory floor with the addition of a very small magnet, approximately $10 \mathrm{~mm}^{3}$ in volume, to one of the rotor

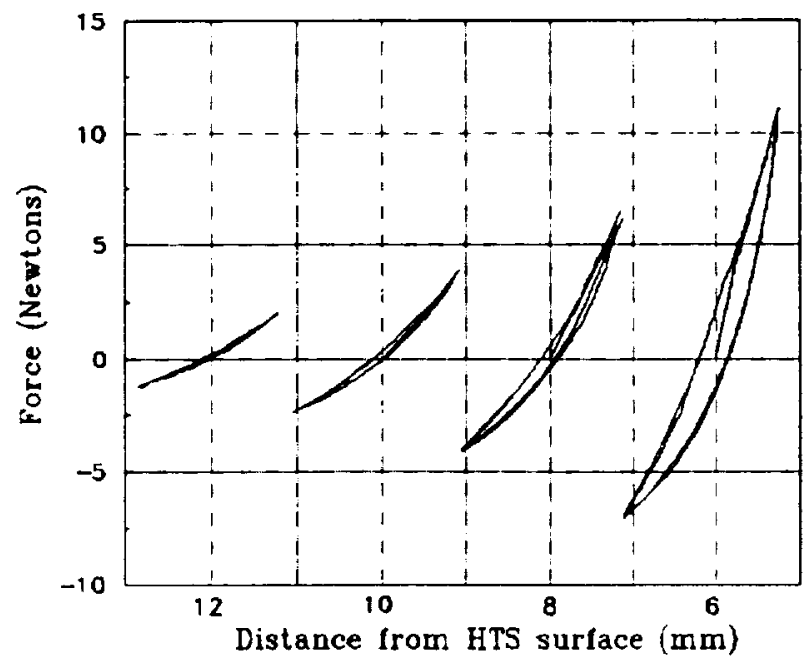

Fig. 4. The field cooled axial force vs axial separation distance between rotor magnet and HTS for field cooling at gaps of $12 \mathrm{~mm}, 10 \mathrm{~mm}, 8 \mathrm{~mm}$, and $6 \mathrm{~mm}$. The initial force here is always zero for the field cooled magnet as is usually the case.

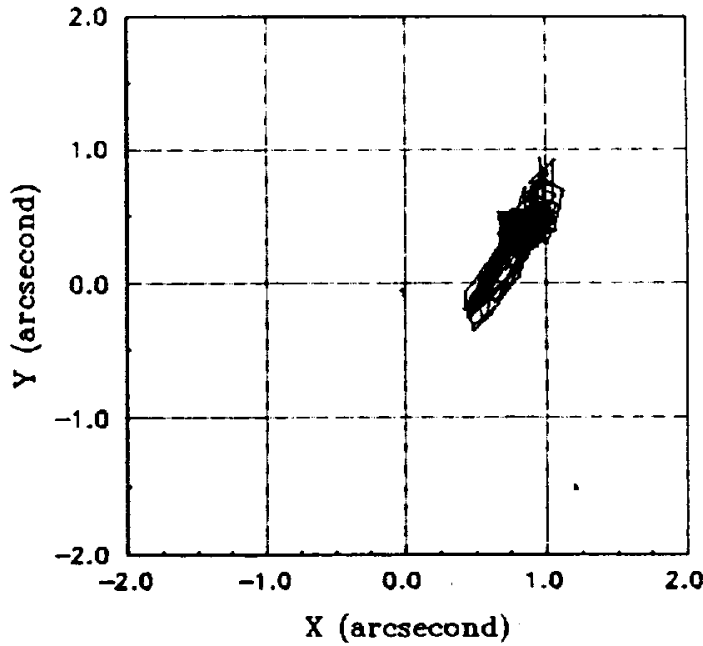

Fig. 5. Trace of reflected laser spot angular position for observation period of approximately 0.5 sec.

magnets on the side facing the HTS. This served to add friction to the bearing to keep the assembly from rotating back and forth during measurement. The entire assembly has been observed to rotate continuously under its own inertia for over $\mathbf{4 5}$ minutes after being hand spun and will rotate back and forth due to small air drafts. Observations on the direction of a laser reflected off a mirror mounted on the platform to an $x-y$ position sensor were made for various time scales. A typical trace for the laser spot is reproduced in Fig. 5. The spot size, measured as the standard deviation of the angular position from the mean, as a function of the total observation period was determined to increase monotonically, from about 0.2 arcseconds after $8 \mathrm{msec}$ of time to 4 arcseconds after $32 \mathrm{sec}$ of time.

\section{CONCLUSIONS}

Before the telescope can track stars, the drive mechanism and its associated control will still have to be developed and integrated into the bearing module. To meet the desired 0.1 arcsecond accuracy under these particular experimental conditions, the control motor mechanism used for tracking would have to be able to respond within a time frame of less than $8 \mathrm{msec}$, which is possible. The dynamic characteristics of the combined HSMB and control motor mechanism will have to be designed accordingly to achieve the results required. This would include consideration of the vibration response of the combined system. The HTS-magnet system shows significant ability to be engineered as a non-contact vibration isolation device [10] and with future refinement of the HSMB design along these considerations promises further improvement in performance.

The bearing module was designed and built in 45 days and has been in periodic use since for over 9 months. Within this time, it has been cycled into operation approximately twenty five times and shipped across the country and has 
proven itself a very simple and robust mechanism. The bearing module can easily be tilted by 45 degrees and still maintain the platform spinning, though the liquid nitrogen spills out. Although developed for use on the moon, these bearings can as well be applied on earth in applications where non-contact levitation and the inherent low friction outweigh the costs of a cryogen. With the HSMB technology, very large weights can be levitated and complications due to material properties can be educed in such a way that design and fabrication is a straightforward process. We hope that these bearings will find themselves useful and be of service in numerous applications, on the moon, in space as well as on earth.

\section{ACKNOWLEDGMENT}

The collaboration and hospitality of R.J. Oliverson at NASA Goddard Space Flight Center, H. Hojaji at Catholic University of America and R.e. Pitts at Computer Sciences Corporation is gratefully acknowledged. A special thanks to the Physics Machine Shop at the University of Houston and Keca Metal Products of Houston for their thorough and timely work.

\section{REFERENCES}

P.C. Chen, R. J. Oliverson, and H. Hojaji, "Advanced Technology Lunar Telescopes," in HTS Makerials, Bulk Processing \& Bulk Applications, Proceedings of the 1992 TCSUH Workshop, Houston, Texas, C.W. Chu, W.K. Chu, P.H. Hor, and K. Salama, Eds. Singapore: World Scientific, 1992, pp. 513-524.
[2] L.J. Payne, R.A. Kimble, A.M. Smith, J.P. Haas, C.C. Sturgell, Jr. R.E. Wentink, J. Carbone, and P.C. Chen, Bull. Am. Astron. Soc., vol 25, pp. 1303, 1993.

[3] P.C. Chen, R.E. Pitts, R.J. Oliversen, J.D. Stolarik, K. Segal, T.L. Wilson, E.I. Lin, J.R. Hull, R. Romero, H. Hojaji, K.B. Ma, Q.Y. Chen, W.K. Chu, and C.W. Chu, Bull. Ain. Astron. Soc., vol 25, pp. $1303,1993$.

[4] C.K. McMichael, K.B. Ma, M.A. Lamb, M.W. Lin. L. Chow, R.L Meng, P.H. Hor, and W.K. Chu, "Practical Adaptation in Bulk Superconducting Magnetic Bearing Applications," Appl Phys. Leth. vol. 60, pp. 1893-1895, April 1992.

[5] D.K. Rao and S.S. Bupra, "Development of a Passive Superconducting Bearing to Support Heavy Rotors at High Speeds," in HTS Materials, Bulk Processing \& Bult Applications. Proceedings of the 1992 TCSUH Workshop, Houston, Texas, C.W. Chu, W.K. Chu, P.H. Hor, and K. Salama, Eds. Singapore: World Scientific, 1992, pp. 436-441.

[6] Q. Chen, K.B. Ma, C.K. McMichael, H. Xia, M.A. Lamb, R.S Cooley, P.C. Fowler, and W.K. Chu, "Designing and Testing of High Tc Superconducting Magnetic Bearing for Flywheel Energy Storage Applications," in Proceedings of the 29th Intersociety Energy Conversion Engineering Conference, vol. 3, pp. 1496, August 1994.

[7] A.N. Terentiev, "Disappearance of Friction in a levilated YBaCuO Superconductor in a Variable Magnetic Field," Physica, vol. 166, pp. $71-74,1990$.

[8] F.C. Moon, P.-Z Chang, H. Hojaji, A. Barkatt and A.N. Thorpe, "Levitation forces, relaxation and magnetic stiffness of meltquenched $\mathrm{YBa}_{2} \mathrm{Cu}_{3} \mathrm{O}_{\mathrm{x}}$," Japanese Joumal of Applied Physics, vol. 29. pp. 1257-1258, July 1990.

[9] A.N. Ierentiev, and A.A. Kuznetsov, "Drift of Levitated YBCO Superconductor Induced by Both a Variable Magnetic Field and a Vibration," Physica C, vol. 195, pp. 41-46, 1992.

[10] M.A. Lamb, K.B. Ma, C.K. McMichael, R.L. Meng, P.H. Hor, R. Weinstein, I. Chen, and W.K. Chu, "Characterization of Non-contact Vibration Absorbers Using YBaCuO," in HTS Materials, Bulk Processing \& Bulk Applications, Proceedings of the 1992 TCSUH Wontshop, Houston, Texas, C.W. Chu, W.K. Chu, P.H. Hor, and K. Salama, Eds. Singapore: World Scientific, 1992, pp. 436-441. 
